\title{
ОЦЕНКА ПРИЧИН СНИЖЕНИЯ ОВАРИАЛЬНОГО РЕЗЕРВА У ЖЕНЩИН РЕПРОДУКТИВНОГО ВОЗРАСТА
}

\section{ASSESSMENT OF THE REASONS OF DECREASING OVARIAL RESERVE IN REPRODUCTIVE AGE WOMEN}

\section{Budanova}

Summary. Object: To conduct a systematic review of the literature on the problem of decreased ovarian reserve in women of reproductive age.

Methods: in order to perform a review of the scientific literature, a collection and analysis of publications in the PubMed text database, the journal of human reproductive sciences, as well as domestic literature on folliculogenesis and the assessment of ovarian reserve was carried out.

Findings: the main reasons for a decrease in ovarian reserve and the main risks of a decreased ovarian reserve, methods of its assessment are described.

Conclusions: research data have been analyzed, the main reasons for a decrease in ovarian reserve are highlighted, the diagnostic role of screening for ovarian reserve may have some prospects in giving an individual woman a better idea of her personal ovarian reserve status, and not just providing her with general advice on age fertility.

Keywords: anti-Müllerian hormone, pool of primordial follicles, in vitro fertilization, low ovarian reserve.

\author{
Буданова Мария Александровна \\ Врач-терапевт, Городская клиническая больнича \\ им. М.П. Кончаловского, г. Москва \\ budan220997@gmail.com
}

Аннотация. Цель исследования (0bject): провести систематический обзор литературных данных по проблеме снижения овариального резерва у женщин репродуктивного возраста.

Методы (Methods): с целью выполнения обзора научной литературы был осуществлён сбор и анализ публикаций в текстовой базе данных PubMed, журнала репродуктивных наук человека, а также отечественной литературе, посвящённой фолликулогенезу и оценке овариального резерва.

Результаты (Findings): описаны основные причины снижения овариального резерва и основные риски возникновения сниженного овариального резерва, методы его оценки.

Выводы (Conclusions): проанализированы данные исследований, выделены основные причины снижения овариального резерва, диагностическая роль скрининга овариального резерва может иметь некоторые перспективы в том, чтобы дать отдельной женщине лучшее представление о ее личном статусе овариального резерва, а не просто предоставить ей общие советы по возрастной фертильности.

Ключевые слова: антимюллеров гормон, пул примордиальных фолликулов, экстракорпоральное оплодотворение, низкий овариальный резерв.

менность до 35 лет [7]. Неудивительно, что это привело к удвоению заболеваемостью бесплодием за последние два десятилетия.

Низкий овариальный резерв является важным ограничивающим фактором для успеха любого метода лечения бесплодия. Об этом свидетельствует снижение количества и качества ооцитов у женщин репродуктивной возрастной группы. Данное явление может находиться в тесной взаимосвязи с возрастом, что, по сути, и наблюдается в позднем репродуктивном периоде. Кроме того, это может встречаться у молодых женщин из-за различных этиологических факторов. В связи с этим оценка овариального резерва яичников, а также индивидуализация терапевтических стратегий очень важны для оптимизации успеха. Большинство женщин со сни- 
женным овариальным резервом вынуждены прийти к процедуре экстракорпорального оплодотворения для достижения беременности. Тем не менее, частота наступления беременности остается низкой, несмотря на множество вмешательств. Раннее выявление и активное наблюдение женщин с низким овариальным резервом обязательны для минимизации необходимости использования донорских яйцеклеток.

\section{Материалы и метолы}

Были проанализированы научные публикации включающие в себя систематические обзоры исследований, проспективные клинические когортные исследования, консенсусные документы и рекомендации ESHRE и ASRM, ретроспективные обсервационные исследования журнала human reproduction в период с 2016 по 2021. Использовалась текстовая база данных медицинских и биологических публикаций PubMed, медицинская библиотека NCBI, журналы Academic Oxford, ESHRE и ASRM, а также отечественные источники: женское бесплодие клинические рекомендации (современные подходы к диагностике и лечению), статьи журнала акушерства и женских болезней, журнала «Акушерство и гинекология».

\section{^итературный обзор}

Под овариальным резервом понимают функциональный резерв яичника, который определяет способность последнего к развитию здорового фолликула с полноценной яйцеклеткой и адекватному ответу на овариальную стимуляцию. Овариальный резерв отражает количество находящихся в яичниках фолликулов (примордиальный пул и растущие фолликулы) и зависит от физиологических и патофизиологических факторов. [3, с.40]

Понятие овариального резерва стоит отличать от фолликулярного запаса, который включает в себя число фолликулов и не отражает их функционального состояния. [2, с. 53] Согласно современным представлениям, выделяют тотальный овариальный резерв, который объединяет в себе фолликулярный запас (количество ооцитов в яичнике) и фолликулогенез (формирование и функционирование доминантного фолликула и желтого тела) [4].

Оценка овариального резерва является обязательной у всех женщин, обращающихся по поводу бесплодия, и определяется с помощью нижеперечисленных параметров:

- возраст женщины;

- количество антральных фолликулов при ультразвуковом исследовании; у уровень антимюллерова гормона;

- уровень фолликулостимулирующего гормона и эстрадиола;

- ответ на стимуляцию гонадотропинами,

- оценка ооцитов и/или эмбрионов во время программы ЭКО либо ИКСИ, (основанная на числе, морфологии или генетической оценке ооцитов). [8]

Итак, разберём подробнее данные пункты.

1. Определение количества антральных фолликулов (КАФ) при трансвагинальном ультразвуковом исследовании (УЗИ) в раннюю фолликулярную фазу цикла.

КАФ представляет собой сумму антральных фолликулов в яичниках в 1-й фазе менструального цикла. Антральные фолликулы определяют, как фолликулы со средним диаметром 3-10 мм в наибольшей двумерной плоскости; низкий овариальный резерв соответствует диапазону от 3 до 5 антральных фолликулов и связан с плохим ответом на овариальную стимуляцию, и низкими шансами наступления беременности [8].

2. Определение гормонов в крови включает: Определение базальной концентрации ФСГ, АМГ, пролактина (ПРЛ), ЛГ, эстрадиола (Е2), общего тестостерона (Т), тиреотропного гормона (ТТГ) и антител к тиреопероксидазе, которое проводится на 2-5-й день фолликулярной фазы цикла.

Концентрация АМГ в крови не зависит от уровня гонадотропинов и является относительно постоянной величиной как у фертильных женщин, так и у женщин с бесплодием, поэтому его можно определять в любой день цикла. Уровень АМГ <1,2 нг/мл ассоциирован с высокой вероятностью «бедного» ответа яичников на стимуляцию, низким качеством эмбрионов и низкими шансами наступления беременности в программах вспомогательных репродуктивных технологий (ВРТ). Уровень АМГ> 3,6 нг/мл связан с чрезмерным ответом яичников и риском развития синдрома гиперстимуляции яичников (СГЯ) в программах ВРТ.

Определение концентрации уровня фолликулостимулирующего гормона (ФСГ) в крови проводится на 2-5 дни менструального цикла. Уровень ФСГ> = 12 ME/л ассоциирован с плохим ответом на стимуляцию яичников и низкими шансами наступления беременности. [8] «Целесообразно одновременно исследовать уровень ФСГ и АМГ; у женщин до 38 лет при оценке гормональных параметров овариального резерва следует обращать внимание как на уровень АМГ, так и на уровень ФСГ, у пациенток старше 38 лет более значимым показателем является базальная концентрация ФСГ» [8]. 
Причины снижения овариального резерва

К основным патологическим факторам, приводящим к снижению овариального резерва, относятся с эндометриоидные кисты яичников, воспалительные заболевания органов малого таза (в частности, хламидиоз, генитальный туберкулез [13]), оперативные вмешательства на яичниках, химиотерапия и радиационное облучение органов малого таза [14], избыточная масса тела [15], курение [16], дефицит витамина Д [21] и др. Далее подробнее разберём каждый из выше перечисленных факторов.

\section{ЭнАометриоиАные кисты яичников}

«Эндометриоз как причина бесплодия занимает второе место после воспалительных заболеваний матки и придатков. До 40\% больных эндометриоидными кистами яичников (ЭКЯ) сталкиваются с проблемами бесплодия. Несмотря на многочисленные исследования, точные механизмы, посредством которых эндометриоз влияет на фертильность, до конца не выяснены. В настоящее время бесплодие у пациенток с (ЭКЯ) наряду с другими значимыми причинами связывают с уменьшением овариального резерва яичников. Существует много механизмов, которые приводят к нарушению овариального резерва при ЭКЯ. Еще в 1957 г. P. Hughesdon и соавт. было описано, что в ткани коркового слоя яичника, располагающейся непосредственно возле ЭКЯ, имеются признаки метаплазии и дезорганизации. Барьер, отделяющий ткань яичника от кисты, состоит из фиброзной ткани. Недавние исследования показали, что эндометриоидная жидкость содержит ряд токсичных веществ, таких как провоспалительные цитокины и активные формы кислорода. Данные вещества вызывают фиброз в окружающих кисту тканях яичника и снижение количества стромальных клеток в корковом слое яичника. Впоследствии фиброз совместно с активными формами кислорода приводит к снижению ангиогенеза, уменьшению плотности капилляров в тканях яичника. Снижение васкуляризации в корковом слое яичника при ЭКЯ может привести к ухудшению кровоснабжения фолликулов и их потере. В корковом слое яичников при эндометриозе происходит ускоренный рост фолликулов с последующим истощением и снижением их количества. Эта теория «выгорания» была предложена M. Dolmans и соавт. в 2007 г. Данная теория была подтверждена в исследованиях M. Kitajiama и соав. Ими было выявлено значительное увеличение процентного содержания первичных фолликулов в корковом слое яичника с ЭКЯ по сравнению с интактным яичником. В данных исследованиях установлено, что в яичниках с эндометриоидными кистами может быть активировано раннее развитие фолликулов, увеличение количества атрезированных фолликулов, нарушение регуляции отбора фолликулов, снижение пула ранних фолликулов, в результате чего исчерпывается пул примордиальных фолликулов. Также группой исследователей высказано предположение, что воспаление и фиброз со сниженной васкуляризацией и повышением оксидативного стресса, которые наблюдаются в корковом слое яичников при эндометриоидных кистах, приводят к нарушению фолликулогенеза. В работах Л.В. Адамян и соавт. было показано нарушение фолликулогенеза яичников женщин с эндометриозом, характеризующееся снижением общего числа фолликулов всех стадий развития. У больных ЭКЯ выявлено резкое снижение числа примордиальных фолликулов, растущих фолликулов. При этом степень уменьшения числа фолликулов в яичниках женщин с эндометриозом зависит от стадии распространения патологического процесса, возраста пациентки. В качестве возможных причин бесплодия при ЭКЯ рассматриваются дистрофические процессы в гранулезных клетках фолликулов, изменение состава фолликулярной жидкости, повышенный апоптотический индекс клеток гранулезы, дегенерация ооцитов. Эндометриоидные клетки секретируют исключительно высокий уровень цитокинов и хемокинов. Цитокины, такие как интерлейкин (ИЛ)-1 $\beta$, ИЛ-6 и фактор некроза опухоли, и хемокины - ИЛ-8, моноцитарный хемоаттрактантный протеин (МСР)-1, могут вызвать активацию клеток гранулезы и преждевременное развитие фолликулов с последующим снижением их количества. Прямо или косвенно эти вещества могут приводить к активации в фолликулярных клетках каскада сигнальных молекул - циклический аденозинмонофосфат протеинкиназы (сAMP/PKA), тирозинкиназа/сигнал-преобразователя и активатора транскрипции (JAK/STAT), ядерного фактора kарра B (NF-кB), фосфатидилинозитол-3-киназы (РІЗК) и внутриклеточного кальция, что приводит к преждевременной активации роста фолликулов. В эндометриоидной ткани в большом количестве производится простагландин Е2 (PGE2), который через аденозинмонофосфат протеинкиназы-а (сAMP/PKA) активирует путь митогенных протеинкиназ (MAPKs), протеин 38 (р38) в ряде типов клеток, включая клетки гранулезы и текаклетки в фолликулах. Можно предположить, что PGE2 и другие эйкозаноиды, образующиеся в воспалительной среде, окружающей эндометриоидную кисту, могут влиять на скорость активации фолликулов и их атрезию. Большинство авторов отмечают более низкие результаты ВРТ у женщин с генитальным эндометриозом в сравнении с другими факторами бесплодия (трубно-перитонеальный, мужской и др.). При этом указывают на худшее качество ооцитов, снижение частоты их оплодотворения и последующего дробления эмбрионов, а также снижение частоты имплантации даже при «малых» формах наружного генитального 
эндометриоза. М. Hull и соавт. отмечают, что у пациенток с эндометриозом яичников нарушаются процессы оплодотворения ооцитов и дробления полученных эмбрионов in vitro. По данным A. Pellicer и соавт., исследование эмбрионов на ранних стадиях развития показало относительно низкое число бластомеров у эмбрионов от пациенток с эндометриозом по сравнению с контролем, а также повышение индекса эмбрионов, остановившихся в развитии в условиях оплодотворения и доимплантационного развития in vitro. В литературе имеются данные о низкой способности к имплантации эмбрионов у женщин с эндометриоз-ассоциированным бесплодием. Предполагают, что причиной неудач при лечении бесплодия с использованием репродуктивных технологий являются неполноценные яйцеклетки, развитие которых происходило в субоптимальных условиях. В работах Л.В. Адамян и соавт. обнаружены не только количественные, но и «качественные» нарушения фолликулогенеза у пациенток с эндометриозом яичников. Так, при гистологическом исследовании фрагментов яичников с эндометриоидными кистами отмечено большое число фолликулов с различными признаками дегенерации (изменение формы и размытые границы фолликула, ооцита и его ядра, фолликулярных клеток; частично деконденсированный хроматин, локализованный по всему ядру; утолщение, расслоение, гиалиноз базальной мембраны фолликула; формирование межклеточных пространств; нечеткая граница между слоями ткани — теки внутренней и наружной; фолликулярная жидкость различной оптической плотности, неравномерно прокрашенная эозином; крупные вакуоли в ооплазме; дезорганизация клеток гранулезы; изменение ширины прозрачной оболочки). Молекулярно-биологические подходы позволили выявить генетические маркеры, указывающие на возможную предрасположенность к эндометриозу. Изучение полиморфизма генов детоксикации ксенобиотиков и метаболизма эстрогенов (цитохрома P450 и глутатионS-трансферазы) позволило обнаружить мутантные аллели этих генов (GSTM 1 0/0, GSTT 1 0/0) у больных эндометриозом в сочетании с медленной формой $\mathrm{N}$-ацетилтрансферазы. При обнаружении у женщин мутации гена эритроцитарной эстеразы $D^{*} 7\left(E S D^{*} 7\right)$ или гена сывороточной эстеразы D*5 (ESD*5) можно предполагать повышенную предрасположенность к эндометриозу яичников в сочетании с эндометриозом брюшины (ESD*7) и к образованию эндометриоидных кист яичников (ESD*5). Таким образом, экспрессия генов, приводящих к развитию эндометриоза, может влиять на фолликулообразование на ранних стадиях формирования яичника. Дефект фолликулогенеза, имеющий место в эмбриональном периоде, в последующем может проявиться как в образовании исходно низкого числа фолликулов, так и в нарушении функции фолликулярных клеток. Данные исследования подтверждают теорию о том, что
ЭКЯ могут привести к повреждению яичников до хирургического вмешательства. При проведении оперативного лечения при ЭКЯ важным аспектом является изучение состояния овариального резерва до оперативного вмешательства. Это позволит оценить степень повреждения ткани яичников до операции, выработать индивидуальную тактику ведения женщин с ЭКЯ и восстановить репродуктивную функцию у данных пациенTOK.» [13]

\section{Оперативные вмешательства}

Выбор хирургических методов лечения эндометриомы (абляция или цистэктомия) может повлиять на послеоперационный овариальный резерв. Одно проспективное рандомизированное исследование показало, что абляция стенки кисты может быть более осуществимо, чем цистэктомия с точки зрения сохранения функции яичников. Другое проспективное рандомизированное исследование с использованием абляции СО2-лазером при лапароскопии второго поколения после орошения и аспирации при первичной лапароскопии и послеоперационной терапии GnRHa в течение 3 мес (трехэтапная процедура) не показало значительного снижения уровня АМГ в послеоперационной сыворотке, в то время как одноэтапная цистэктомия показала значительное снижение уровней АМГ. Другое рандомизированное контролируемое исследование показало, что лапароскопическое удаление эндометриотической кисты яичника значительно снижает уровень АМГ в сыворотке крови и повышает базальные уровни ФСГ независимо от метода, используемого для получения гемостаза на ткани яичника. Недавно исследование, оцениваемое уровнем АМГ после односторонней абляции стенки кисты эндометриомы яичников с использованием энергии плазмы, показало немедленное снижение с последующим прогрессирующим повышением уровня АМГ через несколько месяцев после операции с хорошими исходами фертильности. Этот хирургический метод также показал значительно более высокий послеоперационный КАФ и объем яичников по сравнению с цистэктомией. Эти исследования показывают, что абляционные операции, а не цистэктомия, могут быть осуществимы у женщин, нуждающихся в сохранении овариальной резерва для будущего зачатия.

\section{Аефичит витамина D}

Все более широко признается, что витамин D играет важную роль в репродукции женщин. Недавние исследования показали, что 1a, 25-дигидроксивитамин D3 (VD3), биологически активная форма витамина D, улучшает выживаемость и рост фолликулов яичников in vitro [25]. Для женщин репродуктивного возраста 
уровни 25-гидроксивитамина D3 (кальцифедиола) в сыворотке крови 12,0-20,0 нг / мл и <12,0 нг / мл определяются как недостаточность и дефицит витамина Д соответственно [24].

Во всем мире наблюдается эпидемия дефицита витамина $D$, которая побудила исследовать многие области здоровья и болезней человека. Впоследствии исследования связали дефицит и недостаточность витамина D со многими хроническими заболеваниями сердечно-сосудистой и метаболической систем. Учитывая, что у этого стероидного гормона есть рецепторы по всему телу, включая яичник, в частности, клетки гранулезы, а также в эндометрии и плаценте, очевидно, что витамин D может влиять на репродуктивный успех человека и даже на акушерские исходы.

Существующие на сегодняшний день данные о репродуктивных результатах разнятся. Озкан и др. оценивали фолликулярную жидкость проспективным образом и коррелировали уровни витамина D с исходами беременности. [27] Однако они обнаружили отсутствие корреляции между концентрацией витамина D и параметрами реакции яичников, что позволяет предположить, что эндометрий и восприимчивость эндометрия могут быть ключевыми регуляторами, когда дело доходит до витамина D и репродуктивного успеха. Эта концепция эффекта восприимчивости эндометрия была подтверждена работой Rudick et al. в исследовании модели донорства ооцитов. [28]

Витамин D3 (холекальциферол) вырабатывается в коже или поступает с пищей. 25-гидроксилаза витамина D3, кодируемая полипептидом 1 подсемейства R цитохрома P450 семейства 2 (CYP2R1), превращает витамин D3 в 25-гидроксивитамин D3 (кальцифедиол). Под действием 25-гидроксивитамина D3-1a-гидроксилазы, кодируемой полипептидом 1 подсемейства В цитохрома P450 27 (СYP27B1), 25-гидроксивитамин D3 превращается в 1a, 25-дигидроксивитамин D3 (VD3; кальцитриол). Хотя почка является основным источником циркулирующего VD3, другие органы, включая яичник, также экспрессируют 1a-гидроксилазу, которая может катализировать биосинтез. Следовательно, регуляция функции яичников витамином D может опосредоваться его эндокринным и паракринным действием. VD3 регулирует клеточную функцию через рецептор витамина D (VDR)[30].VDR экспрессируется в различных органах, включая гипоталамус, гипофиз, яичник, яйцевод, матку и плаценту, которые могут изменять свою функцию посредством активации рецепторов к витамину D за счет циркулирующего или локально синтезированного Витамины D3. Таким образом, параметры яичников необходимо оценивать при наличии физиологических уровней VD3, особенно в непатологических условиях, чтобы оценить прямое влияние VD3 на процессы, важные для развития фолликулов, ооцитов, которые подвергаются оплодотворению и последующему эмбриональному развитию.

Влияние витамина D на функцию яичников изучалось в основном в клиническом контексте его влияния на акушерские исходы, особенно у пациентов с нарушениями функции яичников, обращающихся за лечением бесплодия. [29] На сегодняшний день данные о взаимосвязи между концентрацией витамина D в фолликулярной жидкости и частотой наступления беременности противоречивы. Однако добавление витамина D оказывается полезным для женщин, страдающих ожирением и инсулинорезистентностью. Исследования пациентов с синдромом поликистозных яичников показали, что добавление витамина $D$ улучшило рост фолликулов, формирование доминантных фолликулов и частоту наступления беременности, а также способствовало возобновлению и поддержанию менструального цикла [26]. Многоцентровое исследование показало, что дефицит витамина D у женщин позднего репродуктивного возраста (> 40 лет) коррелирует с более сильным снижением резерва яичников [31]. Однако механизмы, с помощью которых витамин D регулирует функцию фолликулов и качество ооцитов, остаются неясными из-за отсутствия адекватных исследовательских моделей.

Предыдущие исследования на животных показали, что самки мышей, соблюдающие диету с дефицитом витамина D после полового созревания, задерживают развитие фолликулов и продлевают эстральные циклы, при этом меньшее количество ооцитов извлекается из яйцеводов после стимуляции гонадотропинами [31]. У самок мышей без рецепторов к витамину D обнаруживалась недостаточность яичников с нарушением развития фолликулов [32]. Биосинтез эстрогенов был нарушен из-за снижения экспрессии генов и активности ароматазы. Похоже, что витамин D необходим для правильной функции яичников и поддержания женской репродуктивной физиологии. Чтобы исключить системные эффекты, вызванные глобальными манипуляциями, например, повышенным уровнем лютеинизирующего гормона (ЛГ) и фолликулостимулирующего гормона (ФСГ) в сыворотке крови у мутантных мышей VDR [32], использовался метод культивирования фолликулов, позволяющий напрямую обрабатывать витамином D фолликулы, полученные in vitro [30]. Данные показали, что добавка витамина D3 улучшила выживаемость фолликулов макака, рост и выработку антимюллерова гормона (АМГ) in vitro.

В настоящем исследовании, вместо того, чтобы охватывать весь период культивирования фолликулов, 
витамин D добавлялся на определенных этапах развития фолликулов для изучения прямого действия VD3 на преантральный и антральный рост и функцию фолликулов, включая секрецию стероидов и созревание ооцитов. Более того, были проведены эксперименты для проверки гипотезы о том, что рецепторы к витамину D и ферменты, важные для биосинтеза витамина D, экспрессируются в фолликулах, развивающихся in vivo и in vitro. Конечные точки также включали эффекты витамина D3 на экспрессию генов, которые имеют решающее значение для передачи сигналов гонадотропина, выработки стероидных и паракринных факторов, а также качества ооцитов.

На эту взаимосвязь, скорее всего, влияет биодоступность витамина D в целевых участках. Связывающий витамин D белок (VDBP) в суперсемействе рецепторов ядерного гормона и альбумин связывают более 99\% доступного активного гормона, и, таким образом, его активность на рецепторе сильно зависит от концентраций VDBP и его полиморфизма, которые могут варьироваться в зависимости от этнической принадлежности. При анализе прямого воздействия на клетки гранулезы активным метаболитом 1,25-дигидроксивитамина D3, который не взаимодействует со связывающим белком, были отмечены изменения в экспрессии гена рецептора антимюллерова гормона (АМГ), а также в передаче сигналов от АМГ далее к органам мишеням. [29]

Эти данные начинают проливать свет на прямые влияния недостаточности витамина D или потенциального механизма действия недостаточности, т.к. это имеет прямое отношение к репродуктивному успеху.

С помощью модели приматов, удалось изучить воздействие 1,25 - дигидроксивитамина D3 на созревание фолликулов от преантральной до антральной стадии. Результаты показали, как 1,25 - дигидроксивитамин D3 влияет на выживание и рост фолликулов, а также на рост ооцитов в зависимости от дозы и стадии. План эксперимента in vitro позволял контролировать, а также подвергать воздействию 1,25-дигидроксивитамина D3 в низких и высоких дозах. Интересно, что низкая доза 1,25-дигидроксивитамина D3 влияла на преантральную выживаемость фолликулов, а высокая доза влияла на диаметр фолликулов. Важно отметить, что больший размер фолликула не обязательно означает более качественный фолликул или ооцит. Стероидные гормоны часто действуют непрерывно, и вполне возможно, что супрафизиологические дозы на самом деле могут иметь меньшую отдачу или даже быть токсичными. [30]

Xu J. Hennebold J.D. Seifer D.B. продемонстрировали как дозозависимый, так и стадийный процесс влияния витамина D на фолликулогенез, что может объяснить некоторые из противоречивых результатов, связанных с влиянием этого стероида на овариальный резерв. Предыдущие данные свидетельствуют о вариабельности концентрации биодоступного витамина D. Эффект дифференциальной дозы 1,25-дигидроксивитамина D3 - низкая доза эффективна на ранней стадии, а высокая доза эффективна позже в фолликулогенезе указывает на потенциальный фактор экспрессии рецептора витамина D, что не было полностью включено в прошлые анализы. [30]

\section{Возраст}

Возраст - важнейший физиологический фактор, который определяет благополучие репродуктивной функции женщины, а также успех лечения бесплодия. Репродуктивная система претерпевает два основных критических периода - половое созревание и наступление менопаузы, характеризующих активацию и прекращение функции яичников.

«К моменту становления менструальной функции количество примордиальных фолликулов в яичниках девочки составляет 270 000-470 000» [11]. В 27-28 лет происходит повышение базального уровня ФСГ, и снижается вероятность зачатия за один менструальный цикл. Проведенные в последние годы исследования $[9,10]$ показали, что скорость исчезновения фолликулов увеличивается в 2 раза, когда примордиальный пул уменьшается до 25000 фолликулов, в норме это соответствует возрасту 37,5 года. Этот возраст определяется как критический, после которого овариальный резерв резко снижается, а к 45 годам вероятность зачатия вовсе приближается к нулю [1]. С каждым менструальным циклом уменьшается общее количество фолликулов.

На сегодняшний день всё больше женщин обращаются к репродуктологам с жалобой на отсутствие беременности в позднем репродуктивном возрасте. У данной возрастной группы, как правило, снижен ОР. Выше уже говорилось, что в 35 лет скорость атрезии фолликулов увеличена в два раза $[1,9,10]$. Возможно, основную роль в снижении фолликулярного запаса яичников играют изменения, происходящие с возрастом в клетках гранулезы, что приводит к уменьшению секреции эстрадиола и ингибина В. Farquhar C.M. и Gleicher N. [1] предположили, что ооциты, заключенные в нерастущие первичные фолликулы не «стареют». Только вступив в процесс созревания, они приобретают риск «потери качества» за счет созревания в измененной околооцитарной среде. Теория старения окружающей среды, а не яйцеклеток предлагает значительные терапевтические возможности в лечении бесплодия в позднем репродуктивном возрасте. 


\section{$\triangle$ ругие}

факторы

Принадлежность к определенной этнической группе также может влиять на овариальный резерв. В исследованиях [14-16] установлено, что женщины из Индии, Юго-Восточной Азии, стран Карибского бассейна, Китая и Латинской Америки имели более низкий овариальный резерв по сравнению с женщинами того же возраста европейской расы и Кавказа.

\section{Ожирение}

В литературе сообщалось о противоречивых результатах исследований, посвященных взаимосвязи между ожирением и овариальным резервом. В исследовании 2019 г. пытались изучить, влияет ли ожирение на сывороточные концентрации маркеров овариального резерва у бесплодных женщин с различным статусом яичникового резерва. В общей сложности 402 женщины были разделены на три группы в соответствии с индексом массы тела (ИМТ <25 кг / м²: нормальный, $\mathrm{n}=$ 198; 25,0-29,9 кг / м²: избыточный вес, $\mathrm{n}=126 ;$ и $\geq 30$ кг / $\mathbf{M}^{2}$ : ожирение, $\left.\mathrm{n}=78\right)$. Женщины также были разделены на две группы по окружности талии (ОТ <80 см: нормальные, $\mathrm{n}=103 ;$ и $\geq 80$ см: страдающие ожирением, $\mathrm{n}=$ 299). Участницы также были разделены на три типа паттернов яичникового резерва: нормальный $(\mathrm{n}=146)$, высокий $(\mathrm{n}=112)$ и плохой овариальный резерв $(\mathrm{n}=144)$. Уровни сывороточного антимюллерова гормона (АМГ), эстрадиола (Е2) и фолликулостимулирующего гормона (ФСГ) были проанализированы и сравнены во всех группах. Подводя итог, не было достоверных различий в ИМТ и ОТ в трех группах яичникового резерва. Были достоверные различия между всеми группами яичникового резерва по АMH, E2 и FSH ( $p<0,001$ для всех). Сравнение параметров овариального резерва (АМГ, Е2 и ФСГ) и параметров ожирения (ИМТ и ОТ) не выявило значимых различий у женщин со всеми типами овариального резерва. [32]

\section{Воспалительные заболевания органов малого таза}

Генитальный туберкулез, даже в латентной форме, всё чаще признается причиной низкого овариального резерва у индийских женщин. Известно, что такие женщины имеют аномальные результаты тестов функциональной диагностики яичников и демонстрируют признаки плохого ответа, требующего высоких доз гонадотропинов для стимуляции яичников при проведении ЭКО. Механизмы, связанные с отсутствием очевидного структурного повреждения, не поняты в настоящий момент. Известно, что "хламидийная инфекция отрицательно влияет на реакцию яичников у тех, кто использует процедуру ЭКО» [11,12,]. Неизбежно склерозирование тканей с нарушением питания фолликулярного аппарата.

\section{ВозАействие химических вешеств и загрязнений}

Химическое загрязнение - это загрязнение окружающей среды, формирующееся в результате изменения ее естественных химических свойств или при поступлении в среду химических веществ, несвойственных ей, а также в концентрациях, превышающих фоновые (естественные) среднемноголетние колебания количеств каких - либо веществ для рассматриваемого периода времени. («Словарь справочник терминов нормативно- технической документации»). Химические загрязнители могут попадать в организм животного и человека напрямую, с водой, ингаляционно при вдыхании. А также попадание вредных химических веществ в организм может происходить опосредовано, через пищу, например, растения, которые выращены в экологически неблагоприятных местах, или рыба, которая выросла в загрязненных человеком озерах или реках. И, наконец, мясо животных, которым либо специально добавляют в пищу химические добавки для ускорения роста или набора массы, либо они сами питаются растительной пищей, произрастающей на загрязненной почве. Таким образом, из-за нерационального землепользования, земледелия и животноводства, человек своими руками совершенствует ряд возможных путей поступления химический загрязнителей в организм. К химическим загрязнителям можно отнести разные вещества, от тяжелых металлов, до органических соединений, источниками которых являются промышленные предприятия, сельскохозяйственные производства и транспорт. Часто ксенобиотики, в частности тяжелые металлы, существуют в природе как различные смеси и взаимодействующие комплексы. От такого разнообразия эффекты влияния на организм в целом очень разнятся в зависимости от большого количества факторов. В последнее время выделяют даже группу - репродуктивных токсикантов. К репродуктивно токсичным тяжелым металлам относят свинец, ртуть, кадмий, медь. В 2010 году Nandi S. изучая влияния тяжелых металлов на развитие ооцитов буйвола и развитие in vitro, показал, что кадмий и свинец оказывают дозазависимое влияние на жизнеспособность, морфологические аномалии, созревание, расщепление и выход бластоцисты. Интоксикации различными химическими веществами, которые используются в промышленности, сельском хозяйстве в качестве пестицидов, гербицидов, растворителей, отходы промышленности (тяжелые металлы и продукты химического синтеза) также могут приводить к снижению овариального резерва $[17,18]$. 


\section{ВывОАы}

Овариальный резерв является важной составляющей частью потенциала фертильности женщины.

1. Для прогнозирования потенциала фертильности необходимо использовать основные методы диагностики овариального резерва: УзИ с целью определения объёма яичника (более $8 \mathrm{~cm}^{3}$ ), количества антральных фолликулов (более 5 в каждом яичнике), измерение уровня антимюллерова гормона (более 1 нг/мл);

2. основными причинами снижения овариального резерва являются: поздний репродуктивный возраст; эндометриоидное поражение яичников; хирургическое лечение путём резекции яичника, эмболизация маточных артерий; ВЗОМТ, радиационное, химиотерапевтическое лечение;

3. несмотря на современные подходы использования органосохраняющих операций при яичниковых образованиях, значительные терапевтические возможности лечения бесплодия в позднем репродуктивном возрасте, вспомогательные репродуктивные технологии (ЭКО, ИКСИ), которые практически не оставляют шанса бесплодию, женщине необходимо задумываться о реализации своей репродуктивной функции до момента снижения работы половых желез, то есть до 2728 лет.

\section{ЛИТЕРАТУРА}

1. Денисенко, М.В. Диагностическое и прогностическое значение состояния ооцит - фолликулярной системы в оценке овариального резерва: дисс. канд. мед. наук. - М., 2018.-9-20 с.

2. Боярский К.Ю. Роль показателей овариального резерва при лечении бесплодия методом ЭКО-ПЭ // Лечение женского и мужского бесплодия (вспомогательные репродуктивные технологии) / под ред. Кулакова В.И., Леонова Б.В., Кузьмичева Л.Н. — М.: МИА, 2005. - С. 53-60.

3. Назаренко Т.А., Смирнова А.А. Индукция моно- и суперовуляции: оценка овариального резерва, ультразвуковой и гормональный мониторинг // Проблемы репродукции. - 2004.— № 1.- С. 39-43

4. Боярский К.Ю. Факторы, определяющие овариальный резерв. Журн. акушерства и женских болезней. 2009. Вып. 2; с. 65-71.

5. Joffe M, Key J, Best N, Jensen TK, Keiding N. The role of biological fertility in predicting family size, Hum Reprod, 2009, vol. 24 (pg. 1999-2006)

6. Mills M, Rindfuss RR, McDonald P, te Velde E. ESHRE Reproduction and Society Task ForceWhy do people postpone parenthood? Reasons and social policy incentives, Hum Reprod Update, 2011, vol. 17 (pg. 848-860)

7. Schmidt L, Sobotka T, Bentzen JG, Nyboe Andersen A. ESHRE Reproduction and Society Task ForceDemographic and medical consequences of the postponement of parenthood, Hum Reprod Update, 2012, vol. 18 (pg. 29-43)

8. Женское бесплодие (современные подходы к диагностике и лечению). Клинические реклмендации (протокол лечения). М3 РФ 05 марта 2019 г. № 15-4/u/2-1913-15c.

9. Pelosi E, Simonsick E, Forabosco A, Garcia-Ortiz J.E, Schlessinger D. Dynamics of the Ovarian Reserve and Impact of Genetic and Epidemiological Factors on Age of Menopause. Biol Reprod. 2015 May;92(5):130. doi: 10.1095/biolreprod.114.127381

10. Ottolenghi C, Uda M, Hamatani T, Crisponi L, Garcia J.E, Ko M, Pilia G, Sforza C, Schlessinger D, Forabosco A. Aging of oocyte, ovary, and human reproduction. Ann N Y Acad Sci. 2004;1034:117-131. doi: 10.1196/annals.1335.015

11. Кузнецова И.В., Уварова Е.В., Коновалов В.А. Проблемы гормональной контрацепции у подростков // Репродуктив. здоровье детей и подростков. 2008. № 6.

12. Кулаков, В.И. Проблемы и перспективы лечения бесплодия в браке/В.И. Кулаков, Овсянникова// Акушерство и гинекология. — 1997. — № 3, — c. 5-8.

13. Современный взгляд на овариальный резерв у женщин репродуктивного возраста с эндометриоидными кистами яичников (обзор литературы) Е.С. Филлипова и соавт.//Проблемы репродукции — 2017, doi: 10.17116/repro201723272-80

14. Iglesias C, Banker M, Mahajan N, Herrero L, Meseguer M, Garcia-Velasco J.A. Ethnicity as a determinant of ovarian reserve: Differences in ovarian aging between Spanish and Indian women. Fertil Steril. 2014 Jul;102(1):244-249. doi: 10.1016/j.fertnstert.2014.03.050

15. Jayaprakasan K, Pandian D, Hopkisson J, Campbell BK, Maalouf WE. Effect of ethnicity on live birth rates after in vitro fertilisation or intracytoplasmic sperm injection treatment. BJOG. 2014 Nov;121(12):300-306. doi: 10.1111/1471-0528.12504

16. Bleil ME, Gregorich SE, Adler NE, Sternfeld B, Rosen MP, Cedars MI. Race/ethnic disparities in reproductive age: An examination of ovarian reserve estimates across four race/ethnic groups of healthy, regularly cycling women. Fertil Steril. 2014 Jan;101(1):199-207.

17. T Var, S Batioglu, E Tonguc, I Kahyaoglu: The effect of laparoscopic ovarian cystectomy versus coagulation in bilateral endometriomas on ovarian reserve as determined by antral follicle count and ovarian volume: a prospective randomized study. Fertil Steril 95, 2247-2250 (2011) D0l: 10.1016/j.fertnstert.2011.03.078

18. D Tsolakidis, G Pados, D Vavilis, D Athanatos, T Tsalikis, A Giannakou, BC Tarlatzis: The impact on ovarian reserve after laparoscopic ovarian cystectomy versus three-stage management in patients with endometriomas: a prospective randomized study. Fertil Steril 94:71-77 (2010) D01: 10.1016/j.fertnstert.2009.01.138

19. S Ferrero, PL Venturini, DJ Gillott, V Remorgida, U Leone Roberti Maggiore: Hemostasis by bipolar coagulation versus suture after surgical stripping of bilateral ovarian endometriomas: a randomized controlled trial. J Minim Invasive Gynecol 19, 722-730 (2012) D0l:10.1016/j.jmig.2012.08.001

20. H Roman, M Bubenheim, M Auber, L Marpeau, L Puscasiu. Antimullerian hormone level and endometrioma ablation using plasma energy. JSLS18, pii: e2014.0.0002 (2014) No DOI found 
21. H Roman, M Auber, C Mokdad, C Martin, A Diguet, L Marpeau, N Bourdel: Ovarian endometrioma ablation using plasma energy versus cystectomy: a step toward better preservation of the ovarian parenchyma in women wishing to conceive. Fertil Steril 96, 1396-1400 (2011) D0I:10.1016/j.fertnstert.2011.09.045

22. Fuhua Xu, Shally Wolf, O'ryai Green, and Jing Xu: Vitamin D in follicular development and oocyte maturation in Reproduction D0I:10.1530/REP-20-0608

23. Franasiak JM, Lara EE \& Pellicer A 2017 Vitamin D in human reproduction. Current Opinion in Obstetrics and Gynecology 29 p.189-194. doi.org/10.1097/ GC0.0000000000000375

24. Zhao G, Ford ES, Tsai J, Li C \& Croft JB2012 Factors associated with vitamin D deficiency and inadequacy among women of childbearing age in the United States. ISRN Obstetrics and Gynecology 2012 691486//doi.org/10.5402/2012/691486

25. Jing Xu, Maralee S Lawson, Fuhua Xu, Yongrui Du, Olena Y Tkachenko, Cecily V: Vitamin D3 Regulates Follicular Development and Intrafollicular Vitamin D Biosynthesis and Signaling in the Primate Ovary 2018, PubMed D0I: 10.3389/fphys.2018.01600

26. Fang, F., Ni, K., Cai, Y., Shang, J., Zhang, X., and Xiong, C. (2017). Effect of vitamin D supplementation on polycystic ovary syndrome: a systematic review and meta-analysis of randomized controlled trials. Complement. Ther. Clin. Pract. 26, 53-60. doi: 10.1016/j.ctcp.2016.11.008

27. Ozkan S. Jindal S. Greenseid K. Shu J. Zeitlian G. Hickmon C.et al. Replete vitamin D stores predict reproductive success following in vitro fertilization. Fertil Steril. 2010; 94: 1314-1319

28. Rudick B. Ingles S.A. Chung K. Stanczyk K. Paulson R.J. Bendikson K.A. Influence of vitamin D levels on in vitro fertilization outcomes in donor-recipient cycles. Fertil Steril. 2014; 101: 447-452

29. Muscogiuri, G., Altieri, B., de Angelis, C., Palomba, S., Pivonello, R., Colao, A., et al. (2017). Shedding new light on female fertility: the role of vitamin D. Rev. Endocr. Metab. Disord. 18, 273-283. doi: 10.1007/s11154-017-9407-2

30. Xu J. Hennebold J.D. Seifer D.B. Direct vitamin D3 actions on rhesus macaque follicles in three-dimensional culture: assessment of follicle survival, growth, steroid, and anti-Mullerian hormone production.Fertil Steril. 2016; 106: 1815-1820

31. Merhi, Z.0., Seifer, D.B., Weedon, J., Adeyemi, 0., Holman, S., Anastos, K., et al. (2012). Circulating vitamin D correlates with serum antimüllerian hormone levels in late-reproductive-aged women: women's interagency HIV study. Fertil. Steril. 98, 228-234. doi: 10.1016/j.fertnstert.2012.03.029

32. Kinuta, K., Tanaka, H., Moriwake, T., Aya, K., Kato, S., and Seino, Y. (2000). Vitamin D is an important factor in estrogen biosynthesis of both female and male gonads. Endocrinology 141, 1317-1324. doi: 10.1210/end0.141.4.7403

33. Obesity Does not Compromise Ovarian Reserve Markers in Infertile Women Umit Gorkem, Ferit Kerim Kucukler, Cihan Togrul, Şebnem Gulen 3 D0l: 10.1055/ a-0650-4723/2019 Jan;79(1):79-85.

( ) Буданова Мария Александровна ( budan220997@gmail.com ).

Журнал «Современная наука: актуальные проблемы теории и практики»

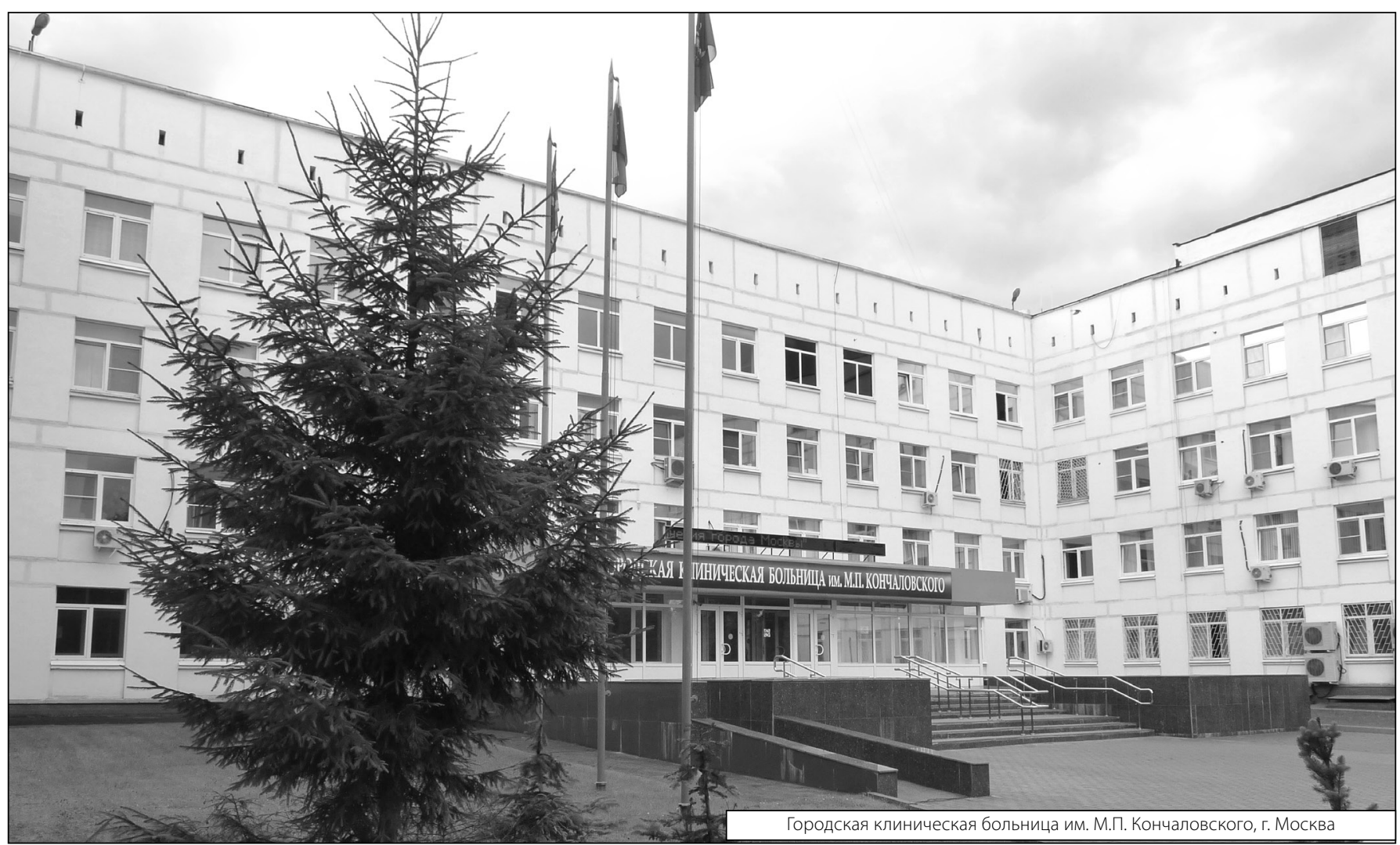

